\title{
Funding and investment decisions in a stochastic defined benefit pension plan with several levels of labor-income earnings th
}

\author{
Ricardo Josa-Fombellidaa ${ }^{\mathrm{a}}$ Juan Pablo Rincón-Zapatero ${ }^{\mathrm{b}, *}$ \\ a Dpto. de Estadística e Investigación Operativa, Universidad de Valladolid, P. Prado de la Magdalena s/n, 47005, Valladolid, Spain \\ ${ }^{\mathrm{b}}$ Dpto. de Economía, Universidad Carlos III de Madrid, C/Madrid 126, 28903 Getafe-Madrid, Spain
}

\begin{abstract}
In this paper we consider the optimal management of an aggregated dynamic pension fund. There are $n$ classes of workers whose salaries are stochastic. A portion of the salary is contributed to the funding process and the manager invests in a portfolio with $m$ risky assets and a risk-free security. The main objective is to minimize the cost of contributions in a bounded horizon $T$ and to maximize the utility of final surplus, measured as the relative fund level respect to the mean salary. The aim of the paper is to describe the properties of fund allocation and optimal contribution when salaries differ across contributors to the fund.
\end{abstract}

Keywords: Finance; Pension funding; Asset allocation; Risk management; Stochastic control

\section{Introduction}

There are two main ways to design a pension fund: the defined benefit pension model, where the benefits are fixed in advance by the sponsor and the contributions are adjusted in order to maintain the fund in balance, and the defined contribution pension model, where contributions are fixed and benefits depend on the fund portfolio. The study of both types of pension plans from the dynamic point of view has been widely discussed in the literature because a great share of the financial operations made in the market by the financial institutions are due to the pension fund activities. See, cf., Boulier et al. [1], Vigna and Haberman [2], Haberman and Vigna [3], Deelstra et al. [4,5], Battocchio and Menoncin [6], in defined-contribution pension funds, Haberman and Sung [7], Chang [8], Haberman et al. [9], Taylor [10], Chang et al. [11] and Josa-Fombellida and Rincón-Zapatero [12,13], in defined-benefit pension funds, and Cairns [14], in both types of plans. These papers use the general problem of optimal portfolio in continuous time initially treated in Merton $[15,16]$.

In this paper we consider a defined benefit stochastic pension plan with random benefits, following a general stochastic process. A deterministic theory contemplating the relevant actuarial variables is given in Bowers et al. [17]. O'Brien $[18,19]$ constitute one of the first attempts to include some randomness elements in the pension plan. Josa-Fombellida

\footnotetext{
${ }_{3}^{\text {th }}$ Both authors gratefully acknowledge financial support from Consejería de Educación y Cultura de la Junta de Castilla y León (Spain) under project VA099/04 and Spanish Ministerio de Ciencia y Tecnología and FEDER funds under project BFM2002-00425. We are indebted to an anonymous referee for the criticisms and valuable comments.

* Corresponding author.

E-mail addresses: ricar@eio.uva.es (R. Josa-Fombellida), jrincon@eco.uc3m.es (J.P. Rincón-Zapatero).
} 
and Rincón-Zapatero [13] provides a different approach, which will be used below. In the present setting, uncertainty comes from salaries evolution.

The most novel feature of our modelling is the existence of $n$ different groups of workers, possibly coming from different companies and having different salaries. All groups are managed as a whole in the same aggregated pension plan, but the manager can recommend a different contribution rate to each group. It is also competence of the manager to decide the quantities invested in the financial assets. However, these investment decisions are aggregated, in the sense that the fund assets are used as a whole in the composition of a mixed portfolio with $m$ risky assets and a riskless bond, and there is no separation by class of workers.

The objective of the manager is to minimize the contributions of the respective groups in this defined benefit plan and, at the date of termination of the plan, to maximize the utility of final surplus, measured as the relative fund level with respect to the mean salary. In this way, our formulation encompasses both the aim of the employer to minimize contributions and the aspirations of the workers of retirement with a guaranteed pension. Note that the final surplus can be distributed between workers that at the finalization of the pension plan have not attained the retirement age. Moreover, the manager can decide to ascribe different importance to the groups in the controlled process. We will consider that the benefits and the contribution rates are proportional to the wages, thus distinguishing each group of workers.

Berkelaar and Kouwenberg [20] considers a retirement saving model, including defined benefit and defined contributions plans as particular cases, in a discrete time setting. The plan is stated for only one individual and, with the obvious particularities, the authors contemplate similar objectives for the controller. As the plan is of non-aggregated type, the payroll benefits are absent except at the final time, which are included in the surplus. One common feature of our framework with the just cited paper is that the wages of the employees are not perfectly correlated with the available assets, so the inherent risk cannot be hedged and the financial market is incomplete. To solve the problem we use dynamic programming techniques, that allows us to find the optimal decisions of investment and contribution payments as functions depending of the state variables, i.e. the level of wealth in the fund and the salaries.

Some authors as Koo [21], Duffie and Zariphopoulou [22] and Duffie et al. [23] have treated an individual investmentconsumption problem with stochastic labor income following a geometric Brownian motion in a continuous time setting on an infinite horizon. These authors investigate the properties of the optimal policies and of the value function, without explicitly solving the problem, due to some computational difficulties appearing because wage income enters in an additive way into the drift of the wealth. Our aggregated defined benefit pension model is not entirely similar to the above problem because salaries have a multiplicative structure in the drift of fund evolution. This fact allows us to find the analytical solution when the salaries are exponential. In fact, as it is shown in Remark 5.1 below, the stochastic control problem appearing in this case can be settled in the classical framework of the Merton model-with $n$ consumptions instead of only one-after some transformations. Hence our main contribution to the literature is not solving analytically the problem in this case, but to carefully study the properties of the solution and the implications from the point of view of fund management.

In the pension funds literature, pension models with stochastic income have been studied by e.g. Cairns et al. [24] and Battocchio and Menoncin [6,25], but in the defined contribution framework. Cairns et al. [24] assumes a random process for salary correlated with the risk-assets and where the objective is to maximize a terminal utility function of the wealth relative to salary. Battocchio and Menoncin [6,25] consider a model with stochastic rate of interest also including the inflation.

In the present paper, we inquire the following important questions: (i) How does the existence of different salaries for the workers influence to the funding process? (ii) What is the optimal portfolio in the presence of random changes in the wages evolution? and (iii) How does the discrimination between classes of workers (i.e. the different weights posed for the manager to the disutility incurred from contributions dedicated to each class of workers) affect the answer of the two first items?

These questions are fairly and completely addressed based on the analytical solution computed under mild restrictions on the elements defining the pension model. Note however, that in the general case we still can extract important information of the optimal funding process. The restrictions alluded above are, on the one hand, the specific choice made of the utility functions and, on the other hand, the assumption of geometric Brownian motions for the salaries. Our main findings in this case are the following: (i) contribution payments are separated for each class of workers, and are equal at every instant of time to the benefits for the retirees at this time minus a percentage of the fund assets which depends on time and also on the weight $\alpha_{i}$ selected for the manager for the $i$ th class of workers. Hence higher levels 
of fund assets lead to a minor contribution, as expected. Another interesting outcome is that the difference between the global benefits and contribution rates (i.e., the sum of all benefits minus the sum of all contributions rates made for all classes of workers) is independent of the salary level, and is a time-variable percentage of fund level; (ii) the random fluctuations of wages modify the usual hedge portfolio appearing in Merton model, that now has an additional term adding to the so-called optimal-growth portfolio strategy. This new term accounts for the correlation between labor earnings and asset returns, in such a way that a high correlation implies investment decisions with lower risk, whereas the opposite is true with low correlation; and (iii) this is a sensitivity analysis question which is easily answered in Section 4 since we know the analytical solution. In general a given group of employees must contribute more than other group when the salary is larger or when the members of the former group have comprised a larger benefit relative to the salary and the sponsor assigns them a lesser weight than to the other group. However, in some cases it is possible to select weights such that a larger salary does not necessarily implies a larger contribution.

The paper is organized as follows. In Section 2 we provide the definitions of the main elements incorporated in a defined-benefit pension plan, considering several groups of workers. Section 3 deals with the statement of the optimization problem, taking care of the two aforementioned objectives of the manager. The different salaries of the workers evolve as rather general processes. In Section 4 we solve the stochastic problem by dynamic programming methods, showing that the value function is separable with respect to the fund and the vector of salaries. Separation of variables then reduces the dimension of the HJB (Hamilton-Jacobi-Bellman) equation. This procedure allows us to obtain some properties of the optimal contribution rates and the hedge portfolio. In particular, they are linear with respect to the fund assets, with factor of proportionality depending on salaries, time and the coefficients defining the control problem. This section finishes with a parametric analysis of the solution, depending on the importance that the sponsor gives to the classes and to the salary size of each class. Section 5 is devoted to the study of a special case where salaries are of exponential type, that is, they are geometric Brownian motions. We observe in this case that we are implicitly using a funding method that is not a spread method, possibly due to the presence of correlation between growth in labor income and asset returns. Section 6 is dedicated to analyzing the particular case when the fund is totally invested in a single risk-free asset. This poses the interesting problem of comparing the size of contributions with those emerging in the mixed portfolio case, as well as comparing the respective terminal fund wealth. We find that in the so-called high salary growth rate case, the strategy of diversification leads to higher mean values of the fund level at the final time but also to a larger contribution effort than in safe investment case. In the so-called moderately growth rate case, we cannot obtain a general conclusion about the fund's level; however, the contribution effort is now less in the diversification framework. Section 7 concludes the paper. All the proofs are in Appendix A.

\section{The pension model}

The pension plan covers $n$ classes of workers, coming from $n$ different labor environments, with the distinctive mean salary characterizing the group. Therefore, it is reasonable to postulate different benefits and contributions rates for each type of worker. Each group is modelled as an aggregated pension plan, with continuous entrance and retirement of members in such a way that the pension population remains stable at every time. The promised liabilities to the workers at the age of retirement are directly related with the mean salary in each class, and the factor of proportionality can vary from class to class. The pension fund has a finite planning horizon $T$. Workers non-retired at this final date receive compensation from the surplus obtained in the process of optimal management of the fund assets. The principal elements intervening in the funding process and the essential hypotheses allowing its temporary evolution to be determined are as follows.

$F(t)$ : Value of fund assets at time $t$.

$B_{i}(t)$ : Benefits promised to the participants of class $i$ at time $t$. They are related with the salary of a worker of this

class at the moment $t$.

$C_{i}(t)$ : Contribution rate in class $i$ at time $t$.

$s_{i}(t)$ : The total salary of the plan members of the class $i$ at time $t$.

$u_{i}(t)$ : The percentage of salary of the class $i$ contributed to plan at time $t$. 
We also consider the vectors $\mathbf{u}=\left(u_{1}, \ldots, u_{n}\right)^{\top}$ and $\mathbf{s}=\left(s_{1}, \ldots, s_{n}\right)^{\top}$, where the symbol ${ }^{\top}$ denotes matrix transposition. We impose the following natural assumption

$$
C_{i}(t)=u_{i}(t) s_{i}(t)
$$

and

$$
B_{i}(t)=k_{i} s_{i}(t)
$$

for $i=1, \ldots, n$, that is, the contributions and the benefits are indexed by the salaries. This is justified on the basis that one of the main concerns of the plan sponsor when promoting a pension fund is to ensure the standard of living of the participants and, unquestionably, this is related with salary. The constants $k_{i}$ are positive and $k_{i} \geqslant u_{i}(t)$ for every $t \in[0, T]$. The total contribution rate and total benefits are

$$
C(t)=\sum_{i=1}^{n} C_{i}(t), \quad B(t)=\sum_{i=1}^{n} B_{i}(t),
$$

respectively. From (1)-(3) the following equality holds

$$
C(t)-B(t)=\sum_{i=1}^{n}\left(u_{i}(t)-k_{i}\right) s_{i}(t) .
$$

For notational convenience, in some places the dependence of the functions on the variables are not shown.

\section{The stochastic control problem}

The randomness involved in financial market is given by two independent standard Brownian motions, one of them $m$-dimensional, $z=\left(z_{1}, \ldots, z_{m}\right)^{\top}$, and the another $d$-dimensional, $w=\left(w_{1}, \ldots, w_{d}\right)^{\top}$, generating the complete probability space $(\Omega, \mathscr{F}, \mathrm{P})$, that is to say, $\mathscr{F}$ is the filtration containing the information available to the sponsor plan, $\left\{\mathscr{F}_{t}\right\}_{t \in[0, T]}$, with $\mathscr{F}_{t}=\sigma\{z(s), w(s) ; 0 \leqslant s \leqslant t\}$.

The plan sponsor manages the fund evolution by means of two instruments:

- The vector of contributions relative to salaries, $\mathbf{u}$, that we suppose a non-negative measurable adapted process with respect to $\left\{\mathscr{F}_{t}\right\}$ satisfying

$$
\int_{0}^{T} \mathbf{u}^{\top}(t) \mathbf{s}(t) \mathrm{d} t<\infty \quad \text { P-a.s. }
$$

- A portfolio built on $m$ risky assets $\left\{P^{i}\right\}_{i=1}^{m}$, which are correlated geometric Brownian motions generated by $z$, and a riskless asset $P^{0}$, whose evolution, as proposed in Merton [15,16], is given by

$$
\begin{aligned}
& \mathrm{d} P^{0}(t)=r P^{0}(t) \mathrm{d} t \quad \text { with } r>0, \\
& \mathrm{~d} P^{i}(t)=P^{i}(t)\left(\mu_{i} \mathrm{~d} t+\sum_{j=1}^{m} \sigma_{i j} \mathrm{~d} z_{j}(t)\right) \quad \text { with } \mu_{i}>0, i=1,2, \ldots, m .
\end{aligned}
$$

It is assumed $\mu_{i}>r$ for all $i$, so the sponsor has incentives to invest with risk. The amount of fund invested at time $t$ in the risky asset $P^{i}$ is denoted by $\lambda_{i}(t), i=1,2, \ldots, m$. The remainder, $F(t)-\sum_{i=1}^{m} \lambda_{i}(t)$, is invested in the bond. Borrowing and shortselling is allowed. ${ }^{1}$ A value $\lambda_{i}$ negative means that the sponsor sells a part of his risky asset $S^{i}$ short while, if $\sum_{i=1}^{m} \lambda_{i}$ is larger than $F$, then he or she gets into debt to purchase the stocks, borrowing at

\footnotetext{
${ }^{1}$ Constraints in borrowing and no shortselling introduce serious complications in the analysis of the problem. A general treatment of the Merton model in this case can be found in Zariphopoulou [26].
} 
rate of interest $r$. We suppose $\{\Lambda(t): t \geqslant 0\}$, with $\Lambda(t)=\left(\lambda_{1}(t), \lambda_{2}(t), \ldots, \lambda_{m}(t)\right)^{\top}$, is a control process adapted to the filtration $\left\{\mathscr{F}_{t}\right\}_{t} \geqslant 0, \mathscr{F}_{t}$-measurable, Markovian and stationary, satisfying

$$
\int_{0}^{T} \Lambda(t)^{\top} \Lambda(t) \mathrm{d} t<\infty, \quad \text { P-a.s. }
$$

We suppose the salary evolution of each class is given by the general stochastic differential equation (SDE)

$$
\mathrm{d} s_{i}(t)=\eta_{i}(\mathbf{s}) \mathrm{d} t+\sum_{j=1}^{m} \beta_{i j}(\mathbf{s}) \mathrm{d} z_{j}(t)+\sum_{j=m+1}^{m+d} \beta_{i j}(\mathbf{s}) \mathrm{d} w_{j-m}(t), \quad i=1, \ldots, n,
$$

with initial conditions $\mathbf{s}(0)=\mathbf{s}^{0}>0$. Moreover obviously we assume $s_{i}>0$ a.s. for all $i$. This is clear, e.g. if salaries are geometric Brownian motions as in Section 5.

Therefore, the fund dynamic evolution under the investment policy $\boldsymbol{\Lambda}$ is

$$
\mathrm{d} F(t)=\sum_{i=1}^{m} \lambda_{i}(t) \frac{\mathrm{d} P^{i}(t)}{P^{i}(t)}+\left(F(t)-\sum_{i=1}^{m} \lambda_{i}(t)\right) \frac{\mathrm{d} P^{0}(t)}{P^{0}(t)}+(C(t)-B(t)) \mathrm{d} t .
$$

By substituting (4), (6) and (7) in (10), we obtain

$$
\mathrm{d} F(t)=\left(r F(t)+\sum_{i=1}^{m} \lambda_{i}(t)\left(\mu_{i}-r\right)+\sum_{i=1}^{n}\left(u_{i}(t)-k_{i}\right) s_{i}(t)\right) \mathrm{d} t+\sum_{i=1}^{m} \sum_{j=1}^{m} \lambda_{i}(t) \sigma_{i j} \mathrm{~d} z_{j}(t),
$$

that, with the initial condition $F(0)=F_{0}>0$ and (9), determines the fund evolution. Next we will assume the notation: $\boldsymbol{\mu}=\left(\mu_{1}, \mu_{2}, \ldots, \mu_{n}\right)^{\top}, \mathbf{1}=(1,1, \ldots, 1)^{\top}, \sigma=\left(\sigma_{i j}\right), \mathbf{k}=\left(k_{1}, k_{2}, \ldots, k_{n}\right)^{\top}$ and $\Sigma=\sigma \sigma^{\top}$. We suppose the existence of $\Sigma^{-1}$, that is, the existence of $\sigma^{-1}$. The vector of standardized risk premia is denoted by $\boldsymbol{\theta}=\sigma^{-1}(\boldsymbol{\mu}-r \mathbf{1})$. Finally, we denote $\boldsymbol{\eta}(\mathbf{s})=\left(\eta_{1}(\mathbf{s}), \ldots, \eta_{n}(\mathbf{s})\right)^{\top}$ and $\beta(\mathbf{s})=\left(\beta^{z}(\mathbf{s}) \mid \beta^{w}(\mathbf{s})\right)$, with $\beta^{z}(\mathbf{s})=\left(\beta_{i j}\right)_{i=1, \ldots, n ; j=1, \ldots, m}$ and $\beta^{w}(\mathbf{s})=$ $\left(\beta_{i j}\right)_{i=1, \ldots, n ; j=m+1, \ldots, m+d}$.

So we can write (9)-(11) in vectorial notation as

$$
\begin{aligned}
\mathrm{d} F(t) & =\left(r F(t)+\Lambda^{\top}(t)(\boldsymbol{\mu}-r \mathbf{1})+(\mathbf{u}-\mathbf{k})^{\top} \mathbf{s}\right) \mathrm{d} t+\boldsymbol{\Lambda}^{\top}(t) \sigma \mathrm{d} z(t), \\
\mathrm{d} \mathbf{s}(t) & =\eta(\mathbf{s}) \mathrm{d} t+\beta^{z}(\mathbf{s}) \mathrm{d} z(t)+\beta^{w}(\mathbf{s}) \mathrm{d} w(t),
\end{aligned}
$$

with initial condition $\left(F_{0}, \mathbf{s}^{0}\right)$.

The plan manager wishes to minimize the contribution payments $\left\{C_{i}\right\}$ of all groups in $[0, T]$. Following the idea of Berkelaar and Kouwenberg [20] for their save-investment model for a single individual, we suppose that higher contributions payments decrease the utility of the manager and increase the marginal utility of reduction in payments. Hence the running utility function, $U(t,-\mathbf{u})$, is increasing and concave with respect to the negative of each of the contribution rates relative to the employees' labor incomes, $-u_{i}$. In this way it is captured the idea that an increase in the contribution rates will probably lead to the plan manager to pay the workers higher salaries in order to hire them originally or to keep them. A family of functions comprising these characteristics is the HARA (hyperbolic absolute risk aversion) type, which furthermore, has the important property of analytical tractability. Specifically, we assume the following specification for the running utility function.

Hypothesis A. The utility function is given by $U(t,-\mathbf{u})=\mathrm{e}^{-\rho t} \prod_{i=1}^{n}\left(k_{i}-u_{i}\right)^{\alpha_{i}}$, where $0<\alpha_{i}<1$ for all $i=1, \ldots, n$, and $\alpha \doteq \sum_{i=1}^{n} \alpha_{i}<1$.

Of course, the instantaneous utility is conveniently discounted at a rate $\rho \geqslant 0$. We can justify the selection of this specific form of the utility from the point of view of game theory. Suppose a hypothetical negotiation process takes place, where the agents are representative workers of each class. Assume that the negotiation concerns the percentage factor of salary to the funding process for each class. If there is no consensus, then the percentage that each class must contribute is the maximum $k_{i}$, hence the disagreement point for agent $i$ is $-k_{i}$, considered as a cost. A bargaining 
game begins with the agents looking for an agreement where the outcome possesses reasonable properties. One of them is Pareto optimality, that states that there is no further possibility of improving the position of one of the classes of workers while leaving the others at least as well off as they were before. The so-called Nash's solution rule has additional properties, like independence of irrelevant alternatives and independence of linear transformations, that is the outcome is the same upon changes in the scale of the utility function. For our problem, denoting $v_{i}=-u_{i}$, the utility function $\prod_{j=1}^{n}\left(v_{j}+k_{j}\right)^{\alpha_{j}}=\prod_{j=1}^{n}\left(v_{j}-\left(-k_{j}\right)\right)^{\alpha_{j}}$ corresponds to the asymmetric Nash bargaining solution, where each player may have different bargaining power, reflected in the exponent $\alpha_{i}$.

Other possible interpretation of the parameters $\alpha_{i}$ is as weighting factors expressing the importance that the manager gives to the contribution made by the $i$ th class of workers to the funding process. They reflect the relative importance for the employer of the different types of salaries. Note that in the case $\alpha_{i}=\alpha / n$, for all $i$, all groups have the same importance.

Additionally, the sponsor's goal is to maximize the utility, $W$, of the final level at fund assets relative to the mean total wage of the groups, $G$. $W$ is an increasing and concave utility function of the form $W(F / G)$. In first place we must define what we understand by total mean salary. We have different possibilities, one of them is to consider the arithmetic mean, $G=\mathbf{1}^{\top} \mathbf{s} / n=\sum_{i=1}^{n} s_{i} / n$. But for analytical convenience we center on a geometric mean with weights given by the relative importance attributed for the manager to each group, $\alpha_{i} / \alpha, i=1, \ldots, n$,

$$
G=G(\mathbf{s})=\left(s_{1}^{\alpha_{1}} s_{2}^{\alpha_{2}} \cdots s_{n}^{\alpha_{n}}\right)^{1 / \alpha},
$$

instead of taking $1 / n$. It can be rewritten as $\ln G=\sum_{i=1}^{n}\left(\alpha_{i} / \alpha\right) \ln s_{i}$, where $\sum_{i=1}^{n}\left(\alpha_{i} / \alpha\right)=1$. For the function $W$ we specify the following form:

Hypothesis B. The final utility function is $W(X)=X^{\alpha}$, with $X=F / G$ and where the parameter $\alpha$ was previously defined, with $0<\alpha<1$.

Thus, given initial values of time, $t_{0}$, fund assets, $F_{0}$, and salaries, $\mathbf{s}^{0}$, the objective functional to be maximized over the class of admissible controls, $\mathscr{A}_{t_{0}, F_{0}, \mathbf{s}^{0}}$, is given by

$$
J\left(\left(t_{0}, F_{0}, \mathbf{s}^{0}\right) ;(\mathbf{u}, \boldsymbol{\Lambda})\right)=E_{t_{0}, F_{0}, \mathbf{s}^{0}}\left\{\int_{t_{0}}^{T} \mathrm{e}^{-\rho\left(t-t_{0}\right)} \prod_{i=1}^{n}\left(k_{i}-u_{i}(t)\right)^{\alpha_{i}} \mathrm{~d} t+\mathrm{e}^{-\rho\left(T-t_{0}\right)}\left(\frac{F(T)}{G(T)}\right)^{\alpha}\right\} .
$$

Here, $\mathscr{A}_{t_{0}, F_{0}, \mathbf{s}^{0}}$ is the set of measurable processes (u, $\boldsymbol{\Lambda}$ ) satisfying (5)-(8) and where $F$ and $\mathbf{s}$ satisfy (12) and (13), respectively. In the above, $E_{t_{0}, F_{0}, \mathbf{s}^{0}}$ denotes conditional expectation with respect to the initial conditions $\left(t_{0}, F_{0}, \mathbf{s}^{0}\right)$. It is important to observe that the imposed admissibility conditions, (5) and (8) guarantee that the system of SDEs defining the fund, (12)-(13), has a unique solution for each initial value of the fund and of the salaries.

Although we have considered a finite horizon for this defined-benefit pension plan, as e.g. in the papers by Haberman and Sung [7] or Berkelaar and Kouwenberg [20], it is straightforward to extend the results to an unbounded horizon planning with the only particularity that the temporary dependence di appear

Notice that when there is a unique group of workers, $n=1$, formally the optimization problem resembles the traditional models of optimal consumption and investment with a single durable consumption good, but without transaction costs, see Grossman and Laroque [27] or Damgaard et al. [28], where the unit price of this good is considered correlated with the financial assets prices, unlike the classic model of Merton [15].

\section{Optimal decisions of contributions and portfolio}

The dynamic programming approach is used to solve the problem. To make the process work, some properties of the value function need to be established. In particular, we prove that it is a solution to the HJB equation. The value function is defined as

$$
\widehat{V}(t, F, \mathbf{s})=\max _{(\mathbf{u}, \boldsymbol{\Lambda}) \in \mathscr{A}_{t, F, \mathbf{s}}}\{J((t, F, \mathbf{s}) ;(\mathbf{u}, \boldsymbol{\Lambda})) \mid \text { s.t. (12), (13) }\} .
$$

It is clear that the value function so defined is non-negative and strictly concave. 
The connection between value functions in optimal control theory (deterministic or stochastic) and optimal feedback controls is accomplished by the HJB equation, see Fleming and Soner [29]. We thus have the following result, where the symbol ${ }^{-\top}$ denotes the transpose of the inverse of a matrix.

Theorem 4.1. Suppose that hypotheses $\mathbf{A}$ and $\mathbf{B}$ hold. Then the optimal relative contribution rates and the optimal investment in the risky assets are given by

$$
\begin{aligned}
& u_{i}(t, F, \mathbf{s})=k_{i}-\frac{\alpha_{i}}{s_{i}} \alpha^{-\gamma} f^{\gamma}(\mathbf{s}) g^{-\gamma}(t, \mathbf{s}) F, \quad i=1, \ldots, n, \\
& \Lambda(t, F, \mathbf{s})=\frac{1}{1-\alpha}\left(\Sigma^{-1}(\boldsymbol{\mu}-r \mathbf{1})+\frac{1}{g(t, \mathbf{s})} \sigma^{-\top}\left(\beta^{z}(\mathbf{s})\right)^{\top} g_{\mathbf{s}}(t, \mathbf{s})\right) F
\end{aligned}
$$

respectively, where $\gamma$ and $f$ are defined by

$$
\begin{gathered}
\gamma=\frac{1}{1-\alpha}, \\
f(\mathbf{s})=\prod_{i=1}^{n}\left(\frac{\alpha_{i}}{s_{i}}\right)^{\alpha_{i}}
\end{gathered}
$$

and where $g:[0, T] \times \mathbb{R}^{n} \longrightarrow \mathbb{R}$ is a positive solution of the non-linear PDE (partial differential equation)

$$
\begin{aligned}
& g_{t}+\left(-\rho+r \alpha+\frac{1}{2} \frac{\alpha}{1-\alpha} \boldsymbol{\theta}^{\top} \boldsymbol{\theta}\right) g+(1-\alpha) \alpha^{-\gamma \alpha} f^{\gamma}(\mathbf{s}) g^{-\gamma \alpha}+\eta(\mathbf{s})^{\top} g_{\mathbf{s}} \\
& \quad-\alpha g_{\mathbf{s}}^{\top} \beta^{z}(\mathbf{s}) \boldsymbol{\theta}+\frac{1}{2} \frac{\alpha}{g} g_{\mathbf{s}}^{\top} \beta^{z}(\mathbf{s})\left(\beta^{z}(\mathbf{s})\right)^{\top} g_{\mathbf{s}}+\frac{1}{2} \operatorname{tr}\left\{\beta(\mathbf{s}) \beta(\mathbf{s})^{\top} g_{\mathbf{s s}}\right\}=0 \quad \forall(t, \mathbf{s}), \\
& g(T, \mathbf{s})=G^{-\alpha}(\mathbf{s}) \quad \forall \mathbf{s} .
\end{aligned}
$$

The optimal relative contribution of group $i, u_{i}$, given by (15) is the difference between the associated relative benefit, $k_{i}$, and a percentage of the fund wealth-income ratio, $F / s_{i}$. Note that this percentage depends also on the remainder salaries. The total contribution rate of the $i$ th salary group presents a similar structure, being the difference between the total benefit and a variable percentage of the fund depending on the whole range of salaries

$$
C_{i}=B_{i}-\alpha_{i} \alpha^{-\gamma} f^{\gamma}(\mathbf{s}) g^{-\gamma}(t, \mathbf{s}) F .
$$

The vector of optimal investment decisions, (16), is an homogeneous linear function of fund level $F$, composed of two terms. The first one contains the investment in the optimal growth portfolio, $\Sigma^{-1}(\mu-r \mathbf{1})$, modified by the constant factor $(1-\alpha)^{-1}$, but the second is a correction term, depending on the correlation parameters between risky assets and salaries and also on time. It is a portfolio that hedges against the unexpected changes in income depending on weights across $g$ but does not on relative benefits. Note that when $\beta^{z}(\mathbf{s})=(0)$, i.e., the growth rate of salary is not correlated with returns on financial assets, the second term is absent.

If we re-write the investments as follows

$$
\Lambda(t, F, \mathbf{s})=\frac{1}{1-\alpha} \sigma^{-\top}\left(\boldsymbol{\theta}+\frac{1}{g(t, \mathbf{s})}\left(\beta^{z}(\mathbf{s})\right)^{\top} g_{\mathbf{s}}(t, \mathbf{s})\right) F,
$$

we observe that the sign of $\lambda_{i}$ depends on the sign and size of the vector function $g_{\mathrm{s}}$, that is to say, of the expressions $\eta(\mathbf{s})$ and $\beta(\mathbf{s})$ determining the salary evolution.

Substituting $\mathbf{u}, \Lambda$, from (15)-(16) into (12), we find that the fund evolution is given by the SDE

$$
\begin{aligned}
\mathrm{d} F(t)= & F(t)\left(\left(r-\alpha\left(\frac{f(\mathbf{s})}{\alpha g(t, \mathbf{s})}\right)^{\gamma}+\frac{1}{1-\alpha}\left(\boldsymbol{\theta}^{\top} \boldsymbol{\theta}+\frac{1}{g(t, \mathbf{s})} g_{\mathbf{s}}^{\top}(t, \mathbf{s}) \beta^{z}(\mathbf{s}) \boldsymbol{\theta}\right)\right) \mathrm{d} t\right. \\
& \left.+\frac{1}{1-\alpha}\left(\boldsymbol{\theta}^{\top}+\frac{1}{g(t, \mathbf{s})} g_{\mathbf{s}}^{\top}(t, \mathbf{s}) \beta^{z}(\mathbf{s})\right)\right) \mathrm{d} z(t),
\end{aligned}
$$


where s satisfies (13). In this way, the SDE satisfied by the fund level is coupled with the SDE of the salaries, making a quite difficult task to exactly integrate this system of SDEs. To go some steps further in the analysis of our model we will proceed to be more specific about the nature of salaries in the next section, supposing that they are geometric Brownian motions.

Turning to the general case, we still can study some qualitative properties without resorting to analytical solution of the problem. An important issue is to address the question of how the optimal contributions of the different groups selected by the manager is influenced by the size of respective comprised benefits. For instance, if we consider the classes $i, j$, taking into account (15) and (21) it is obvious that

(i) If $k_{i} \geqslant k_{j}$ and $\alpha_{i} s_{j} \leqslant \alpha_{j} s_{i}$ then $u_{i} \geqslant u_{j}$.

(ii) If $k_{i} s_{i} \geqslant k_{j} s_{j}$ and $\alpha_{i} \leqslant \alpha_{j}$ then $C_{i} \geqslant C_{j}$.

(i) affirms that if the percentage of salary promised to the representative worker of group $i$ is higher than the corresponding to the worker of group $j$, then the percentage of salary devoted to contribution by group $i$ is higher than that of group $j$, whenever $\alpha_{i} s_{j} \leqslant \alpha_{j} s_{i}$ holds, that is, the relative importance for the manager of the groups-or their bargaining power, as explained above-is lesser than the relative value of the salaries, $\alpha_{i} / \alpha_{j}<s_{i} / s_{j}$. In the case where the groups are attached the same importance for the manager, this simply means that higher contributions are needed to attain higher benefits, which is a reasonable outcome, (ii) establish a similar property as above but for size of the contributions. Now if the total benefits that the workers $i$ will attain at the time of retirement is higher than that of group $j$, and if $\alpha_{i} \leqslant \alpha_{j}$, then contribution are in the same direction. Note carefully that reversing the inequality between $\alpha_{i}$ and $\alpha_{j}$ can lead to a different outcome. Again the equal importance situation leads to a reasonable conclusion.

Consider now the possibility that the maximum percentage of salary devoted to benefit is the same for every group of workers, that is, $k_{1}=\cdots=k_{n}$. Let us arrange the different salaries in a decreasing sequence $s_{1} \geqslant s_{2} \geqslant \cdots \geqslant s_{n}$. A sufficient condition assuring that the contributions satisfy $C_{1} \geqslant C_{2} \geqslant \cdots \geqslant C_{n}$, is that the manager selects weights in the opposite direction, $\alpha_{1} \leqslant \cdots \leqslant \alpha_{n}$. In particular, in the equality case workers with high salaries contribute more to the fund than those with low salaries.

Looking more in deep the case where $\alpha_{1}=\cdots=\alpha_{n}$, we obtain from (21) the remarkable fact that it is optimal that the difference between benefits and contributions at every instant of time is the same for each class of worker. Concretely,

$$
B_{i}-C_{i}=\frac{\alpha}{n}\left(\frac{f(\mathbf{s})}{\alpha g(t, \mathbf{s})}\right)^{\gamma} F, \quad i=1, \ldots, n .
$$

The gap depends directly of the fund level, rising as it increases, and tightening as $F$ goes down. The proportionality factor depends on the several parameters considered—including the number of groups into the plan-and on the different salaries of the workers.

\section{Exponential salaries}

In order to solve Eq. (19) analytically and to have a more near view of the problem we give a specification of the salary processes:

Hypothesis C. Each salary process satisfies

$$
\begin{gathered}
\eta_{i}(\mathbf{s})=\eta_{i}\left(s_{1}, \ldots, s_{n}\right)=\eta_{i} s_{i}, \\
\beta_{i j}(\mathbf{s})=\beta_{i j}\left(s_{1}, \ldots, s_{n}\right)=\beta_{i j} s_{i},
\end{gathered}
$$

with $\eta_{i}, \beta_{i j} \in \mathbb{R}^{+}$for all $i=1, \ldots, n$ and for all $j=1, \ldots, m+d$. We suppose $\eta_{i} \geqslant r$ for all $i$, that is to say, the salary growth is at least the market risk free rate of interest. 
The previous assumption join with (9) imply the next facts:

- Each class salary $s_{i}$ is a geometric Brownian motion with SDE

$$
\mathrm{d} s_{i}(t)=s_{i}(t)\left(\eta_{i} \mathrm{~d} t+\sum_{j=1}^{m} \beta_{i j} \mathrm{~d} z_{j}(t)+\sum_{j=m+1}^{m+d} \beta_{i j} \mathrm{~d} w_{j-m}(t)\right),
$$

with $s_{i}(0)=s^{0}>0$, for $i=1, \ldots, n$. In the following we will employ the same notation $\eta$, $\beta$, eliminating the dependence with respect to $\mathbf{s}$.

- The geometric mean salary $G(\mathbf{s})$ is a geometric Brownian motion with SDE

$$
\begin{aligned}
\mathrm{d} G(\mathbf{s}(t))= & G(\mathbf{s}(t))\left(\left(\sum_{i=1}^{n} \frac{\alpha_{i}}{\alpha} \eta_{i}+\frac{1}{2} \sum_{i, j=1}^{n} \frac{\alpha_{i}}{\alpha} \frac{\alpha_{j}}{\alpha} \sum_{l=1}^{m+d} \beta_{i l} \beta_{j l}-\frac{1}{2} \sum_{i=1}^{n} \frac{\alpha_{i}}{\alpha} \sum_{l=1}^{m+d} \beta_{i l}^{2}\right) \mathrm{d} t\right. \\
& \left.+\sum_{i=1}^{n} \frac{\alpha_{i}}{\alpha}\left(\beta_{i 1}, \ldots, \beta_{i m}\right) \mathrm{d} z(t)+\sum_{i=1}^{n} \frac{\alpha_{i}}{\alpha}\left(\beta_{i, m+1}, \ldots, \beta_{i, m+d}\right) \mathrm{d} w(t)\right)
\end{aligned}
$$

with initial condition $G\left(\mathbf{s}^{0}\right)$. In order to arrive to this equation we have used the Itô's formula with the function $G\left(s_{1}, \ldots, s_{n}\right)=s_{1}^{\alpha_{1} / \alpha} \ldots s_{n}^{\alpha_{n} / \alpha}$.

Another consequence of Hypothesis $\mathbf{C}$ is given in the following result.

Proposition 5.1. Under conditions of Theorem 4.1 and Hypothesis $\mathbf{C}$ the function $g$ is given by

$$
g(t, \mathbf{s})=\alpha^{-\alpha} h^{1-\alpha}(t) f(\mathbf{s}), \quad t \in[0, T], \mathbf{s} \in \mathbb{R}_{+}^{n},
$$

with $h: \mathbb{R} \longrightarrow \mathbb{R}$ the function which expression is

$$
h(t)= \begin{cases}\left(\varepsilon^{-\gamma}+\frac{1-\alpha}{A}\right) \mathrm{e}^{A \gamma(T-t)}-\frac{1-\alpha}{A} & \text { if } A \neq 0, \\ \varepsilon^{-\gamma}+T-t & \text { if } A=0,\end{cases}
$$

for all $t \in[0, T]$, where the parameter $\varepsilon$ is

$$
\varepsilon=\prod_{i=1}^{n}\left(\frac{\alpha_{i}}{\alpha}\right)^{\alpha_{i}}
$$

the constant $A$ is given by

$$
\begin{aligned}
A= & -\rho+r \alpha+\frac{1}{2} \frac{\alpha}{1-\alpha} \boldsymbol{\theta}^{\top} \boldsymbol{\theta}-\sum_{i=1}^{n} \eta_{i} \alpha_{i}+\alpha \sum_{i=1}^{m} \sum_{j=1}^{n} \beta_{j i} \alpha_{j} \theta_{i} \\
& +\frac{1}{2} \alpha \sum_{i=1}^{m}\left(\sum_{j=1}^{n} \beta_{j i} \alpha_{j}\right)^{2}+\frac{1}{2} \sum_{i, j=1}^{n} \sum_{l=1}^{m+d} \beta_{i l} \beta_{j l} \alpha_{i} \alpha_{j}+\frac{1}{2} \sum_{i=1}^{n} \sum_{l=1}^{m+d} \beta_{i l}^{2} \alpha_{i}
\end{aligned}
$$

and $f$ is given by (18).

The previous proposition shows that function $g$ can be separated as the product of a constant, $\alpha^{-\alpha}$, a function depending only on time, $h^{1-\alpha}$, and another function depending only on salaries, $f$. The parameter $A$ depends in a rather complicated way of the coefficients defining the problem.

The assumption of exponential salaries allows us to analytically solve the non-linear PDE (19) with initial condition (20) and finding the expression for $g$. Particularizing Theorem 4.1 to this case we can establish the following conclusions about optimal policies. 
Proposition 5.2. Suppose that Hypotheses $\mathbf{A}, \mathbf{B}$ and $\mathbf{C}$ hold. Then the optimal relative contribution rates and the optimal investment in the risky assets are given by

$$
\begin{aligned}
& u_{i}(t, F, \mathbf{s})=k_{i}-\frac{\alpha_{i}}{\alpha h(t)} \frac{F}{s_{i}}, \quad i=1, \ldots, n, \\
& \Lambda(t, F, \mathbf{s})=\frac{1}{1-\alpha}\left(\Sigma^{-1}(\boldsymbol{\mu}-r \mathbf{1})-\sigma^{-\top}\left(\beta^{z}\right)^{\top} \boldsymbol{\alpha}\right) F,
\end{aligned}
$$

respectively, where $h$ is given in (24) and $\alpha$ is defined by $\alpha=\left(\alpha_{1}, \ldots, \alpha_{n}\right)^{\top}$.

Remark 5.1. It is possible to solve the problem using standard martingale techniques. ${ }^{2}$ We sketch here the general idea because it clarifies the structure of the pension model. Introduce new control variables $c_{i}=-\left(u_{i}-k_{i}\right) s_{i}, i=1, \ldots, n$, instead of the originals, $u_{i}$. Then (14) can be rewritten as

$$
E_{t_{0}, F_{0}, \mathbf{s}^{0}}\left\{\int_{t_{0}}^{T} \mathrm{e}^{-\rho\left(t-t_{0}\right)} \prod_{i=1}^{n} c_{i}(t)^{\alpha_{i}} \prod_{i=1}^{n} s_{i}(t)^{-\alpha_{i}} \mathrm{~d} t+\mathrm{e}^{-\rho\left(T-t_{0}\right)} F(T)^{\alpha} G(T)^{-\alpha}\right\}
$$

and (12) as

$$
\mathrm{d} F(t)=\left(r F(t)+\boldsymbol{\Lambda}^{\top}(t)(\boldsymbol{\mu}-r \mathbf{1})-\mathbf{1}^{\top} \mathbf{c}(t)\right) \mathrm{d} t+\Lambda^{\top}(t) \sigma \mathrm{d} z(t),
$$

with $\boldsymbol{c}=\left(\boldsymbol{c}_{1}, \ldots, \boldsymbol{c}_{n}\right)^{\top}$ using (22), it is easy to compute an expression for $G(\mathbf{s}(t))^{-\alpha}$

$$
G(\mathbf{s}(t))^{-\alpha}=G(\mathbf{s}(0))^{-\alpha} \exp \left\{\boldsymbol{\alpha}^{\top}\left(-\boldsymbol{\eta}+\frac{1}{2} \boldsymbol{v}\right) t-\boldsymbol{\alpha}^{\top}\left(\beta^{z} z(t)+\beta^{w} w(t)\right)\right\},
$$

where $\boldsymbol{v}=\left(v_{1}, \ldots, v_{n}\right)^{\top}, v_{i}=\mathbf{e}_{i}^{\top} \beta \beta^{\top} \mathbf{e}_{i}, \mathbf{e}_{i}=(0, \ldots, 1, \ldots, 0)^{\top}, i=1, \ldots, m+d$. Define now the exponential martingale

$$
M(t)=\exp \left\{-\frac{1}{2} \boldsymbol{\alpha}^{\top} \beta \beta^{\top} \boldsymbol{\alpha} t-\boldsymbol{\alpha}^{\top}\left(\beta^{z} z(t)+\beta^{w} w(t)\right)\right\},
$$

which has expectation equal to one and hence it is a change of probability measure. Consider a measure $H$ with

$$
\frac{\mathrm{d} H}{\mathrm{dP}}=M(T) \quad \text { on }(\Omega, \mathscr{F}, P) \times[0, T]
$$

and $E^{H}$ the expectation with respect to this measure. Then the optimization problem becomes

$$
\max _{\mathbf{c}, \boldsymbol{A}} E_{t_{0}, F_{0}, \mathbf{s}^{0}}^{H}\left\{\int_{t_{0}}^{T} \mathrm{e}^{-\rho^{*}\left(t-t_{0}\right)} \prod_{i=1}^{n} c_{i}(t)^{\alpha_{i}} \mathrm{~d} t+\mathrm{e}^{-\rho\left(T-t_{0}\right)} F(T)^{\alpha}\right\},
$$

with a suitable modification of the discount factor to $\rho^{*}=\rho-\boldsymbol{\alpha}^{\top}\left(-\eta+\frac{1}{2}\left(\boldsymbol{v}+\beta \beta^{\top} \boldsymbol{\alpha}\right)\right)$. Under measure $H$, the process

$$
\left(\begin{array}{c}
z^{H}(t) \\
w^{H}(t)
\end{array}\right)=\left(\begin{array}{c}
z(t)+\left(\beta^{z}\right)^{\top} \boldsymbol{\alpha} t \\
w(t)+\left(\beta^{w}\right)^{\top} \boldsymbol{\alpha} t
\end{array}\right)
$$

is a Brownian motion. The fund evolves then according to

$$
\mathrm{d} F(t)=\left(r F(t)+\boldsymbol{\Lambda}^{\top}(t)(\boldsymbol{\mu}-r \mathbf{1})-\mathbf{1}^{\top} \mathbf{c}(t)\right) \mathrm{d} t+\boldsymbol{\Lambda}^{\top}(t) \sigma\left(\mathrm{d} z^{H}(t)-\left(\beta^{z}\right)^{\top} \boldsymbol{\alpha}\right)
$$

or

$$
\mathrm{d} F(t)=\left(r F(t)+\boldsymbol{\Lambda}^{\top}(t)\left(\boldsymbol{\mu}-r \mathbf{1}-\sigma\left(\beta^{z}\right)^{\top} \boldsymbol{\alpha}\right)-\mathbf{1}^{\top} \mathbf{c}(t)\right) \mathrm{d} t+\boldsymbol{\Lambda}^{\top}(t) \sigma \mathrm{d} z^{H}(t),
$$

and the stock price processes are changed accordingly, obtaining in this way the market price of risk under $H, \theta^{*}=$ $\sigma^{-1}(\boldsymbol{\mu}-r \mathbf{1})-\left(\beta^{z}\right)^{\top} \boldsymbol{\alpha}$. Observe that the problem is now that of Merton with $n$ consumption variables. The change of

\footnotetext{
${ }^{2}$ We are grateful to an anonymous referee for showing us how to reformulate the problem in the exponential salaries case to a Merton type-model with modified discount rate and modified market price of risk. We have preferred to maintain the HJB approach because it is not apparent if the martingale techniques can be used to solve the general problem, and although the HJB equation cannot either be solved, we still can extract some useful consequences of the structure of the solution.
} 
measure and the modification of the discount factor and the market price of risk settle our model in a complete market framework. It is worth noting that the market price of risk is modified to take into account the correlation between asset prices and salaries, $\sigma\left(\beta^{z}\right)^{\top} \boldsymbol{\alpha}$. Note also that in $\rho^{*}$ enters the mean growth rate and the diffusion terms of the salaries. Now applying the results from Merton [16], the optimal portfolio is given by

$$
\boldsymbol{\Lambda}=\frac{1}{1-\alpha}\left(\Sigma^{-1}(\boldsymbol{\mu}-r \mathbf{1})-\sigma^{-\top}\left(\beta^{z}\right)^{\top} \boldsymbol{\alpha}\right) F .
$$

The optimal contributions could be obtained with the usual Lagrangian approach, that can be consulted in e.g. Duffie [30] or Karatzas and Shreve [31].

The observations made about the content of Proposition 4.1 takes here a more specific form. At every instant of time the percentage of salary devoted to the funding process for every group of workers is the difference between the relative benefit, $k_{i}$, and a given percentage of the fund's wealth-income ratio for group $i$. The proportionality constant at every instant of time depends on the weights assigned by the manager to the different groups and of the remainder parameters through function $h$ and does not depend on the salaries $\mathbf{s}$.

Form (26) the contributions are now given by

$$
C_{i}=B_{i}-\frac{\alpha_{i}}{\alpha h(t)} F, \quad i=1, \ldots, n,
$$

hence the difference between benefits and contributions does not depends directly on salaries, and difference between total benefits and total contributions is

$$
B-C=\frac{1}{h(t)} F,
$$

that is, at every instant of time the gap between the total benefits paid to the retired workers and the total contributions made to the funding process by the active members is a positive quantity, ${ }^{3}$ given by a percentage of fund's level. This percentage increases or decreases along time-but always in a monotonic fashion-depending on $h$ and at the date of termination is $\varepsilon^{\gamma} \%$ of the fund assets $F(T)$. The behavior of $h$ depends to a great extent of the size and sign of $A$, properties that accounts for the surplus of the mean salary growth with respect to the market rate of interest and also for the size of the uncertainty in the wages and risky asset prices and their correlation.

The following are straightforward properties of ${ }^{4} h$.

- $h$ is strictly increasing when $A<-(1-\alpha) \varepsilon^{\gamma}$.

- $h$ is strictly decreasing when $0 \geqslant A>-(1-\alpha) \varepsilon^{\gamma}$.

We call the former situation the "high salary growth case" - because in order for $A$ to be more negative than $-(1-\alpha) \varepsilon^{\gamma}$, $\eta_{i}$ must be significatively higher than $r$-whereas the latter is called the "moderate salary growth case" 5 In the first case the relative effort made to the funding process is declining along time; since benefits are indexed by salaries, to assure the solvency of the fund the contributions are a larger proportion of fund's level at the initial dates than at termination. The opposite is true in the second case. An important implication from the point of view of the application of the theoretical results contained in the paper is that the global design of the scheme of contributions respond to a dichotomous situation. For high salary growth the relative effort of contribution is lowering along time, but with salaries with moderate growth that relative effort is progressively increasing.

With respect to the investment portfolio, it now depends only on fund's level—in a linear way — and not on time or salaries. As in the general case analyzed in the above section, to the term appearing in the classical Merton's model we must add a second term which cares about the existing correlation between salaries and asset prices. Depending on the

\footnotetext{
${ }^{3}$ As we can see from formula (29) below, the fund process is in this case a geometric Brownian motion and hence it remains positive if the initial fund level, $F_{0}$, is.

${ }^{4}$ We consider that the equality $A=-(1-\alpha) \varepsilon^{\gamma}$ is anecdotic; in this case $h$ is constant.

${ }^{5}$ Actually, this denomination is not entirely correct because in the definition of $A$ besides the rate growth of salaries, the different sources of uncertainty and their correlation play an important role. Hence it would be more appropriate to talk of the salary growth relatively to the size of the uncertainty.
} 
parameters values of the model, the optimal investment policy can require shortselling of the risky assets or borrowing to invest with risk. The investment decisions are also conditioned by the weights put for the manager to each group. It is necessary to assume more risk, investing in risky assets, when the coefficient of correlation $\beta^{z}$ or the weights $\alpha_{i}$ are smaller. In fact, when $\mathbf{s}$ is uncorrelated with the risky assets, more risk is assumed.

Stochastic salaries lead to a simple evolution of the fund actives, which now obey the SDE

$$
\mathrm{d} F(t)=F(t)\left(\left(r-\frac{1}{h(t)}+\frac{1}{1-\alpha}\left(\boldsymbol{\theta}^{\top} \boldsymbol{\theta}-\boldsymbol{\alpha}^{\top} \beta^{z} \boldsymbol{\theta}\right)\right) \mathrm{d} t+\frac{1}{1-\alpha}\left(\boldsymbol{\theta}^{\top}-\boldsymbol{\alpha}^{\top} \beta^{z}\right) \mathrm{d} z(t)\right),
$$

with $F(0)=F_{0}$. Thus the fund process is uncoupled with salaries and in fact it is a geometric Brownian motion. It is possible to obtain the expected value of fund at final instant $T$. By Arnold [32, p. 140],

$$
E_{F_{0}} F(T)=F_{0} \exp \left\{\left(r+\frac{1}{1-\alpha}\left(\boldsymbol{\theta}^{\top} \boldsymbol{\theta}-\boldsymbol{\alpha}^{\top} \beta^{z} \boldsymbol{\theta}\right)\right) T-\int_{0}^{T} \frac{1}{h(t)} \mathrm{d} t\right\} .
$$

From (28) the expectation of the difference between total benefits and total contribution at final time $T$ is

$$
E_{F_{0}}\{B(T)-C(T)\}=\frac{1}{h(T)} E_{F_{0}} F(T)=\varepsilon^{\gamma} E_{F_{0}} F(T),
$$

where $E_{F_{0}} F(T)$ is given by (30).

Denoting by $\Gamma$ the cumulative income process,

$$
\Gamma(t)=F_{0}-\int_{0}^{t}(B(s)-C(s)) \mathrm{d} s,
$$

with $t \in[0, T]$, the expected cumulative final income is

$$
E_{F_{0}} \Gamma(T)=F_{0}-\int_{0}^{T} \frac{1}{h(t)} E_{F_{0}} F(t) \mathrm{d} t
$$

where $E_{F_{0}} F(t)$ is given by (30), substituting $T$ by $t$.

Remark 5.2. Hypothesis $\mathbf{C}$ assures that the total benefits of class $i, B_{i}$, is a geometric Brownian motion with the same parameters as the corresponding total salary of this class, $s_{i}$, because $B_{i}=k_{i} s_{i}$, with $k_{i}$ constant, for all $i=1, \ldots, n$. Following Josa-Fombellida and Rincón-Zapatero [13], the relationship

$$
\left(\delta-\eta_{i}\right) A L_{i}(t)+N C_{i}(t)=B_{i}(t), \quad t \in[0, T],
$$

is satisfied, for all $i=1, \ldots, n$. In the above the following notation is being used:

$\delta$ : Constant rate of valuation of the liabilities, which can be specified by the regulatory authorities.

$A L_{i}(t)$ : Actuarial liability at time $t$ in class $i$.

$N C_{i}(t)$ : Normal cost at time $t$ in class $i$.

We will also use:

$A L(t)$ : Actuarial liability at time $t$, that is, total liabilities of the sponsor.

$U A L(t)$ : Unfunded actuarial liability at time $t$, equal to $A L(t)-F(t)$.

$N C(t)$ : Normal cost at time $t$. If the fund assets match the actuarial liability, and if there are no uncertain elements in the plan, the normal cost is the value of the contributions allowing equality between asset funds and obligations.

$S C(t)$ : Supplementary cost at time $t$, equal to $C(t)-N C(t)$.

The total rate of contribution in group $i$ is

$$
C_{i}=u_{i} s_{i}=B_{i}-\frac{\alpha_{i}}{\alpha} \frac{F}{h}
$$


for all $i, t$. Taking into account (32),

$$
C_{i}-N C_{i}=\left(\delta-\eta_{i}\right) A L_{i}-\frac{\alpha_{i}}{\alpha} \frac{F}{h}
$$

and summing in all classes,

$$
S C=C-N C=\delta A L-\sum_{i=1}^{n} \eta_{i} A L_{i}-\frac{F}{h}=\frac{1}{h(t)} U A L+\sum_{i=1}^{n}\left(\delta-\eta_{i}-\frac{1}{h(t)}\right) A L_{i} .
$$

Thus the optimal funding process uses an amortization method a bit different to the habitual method registered in the actuarial literature named spread method; see Bowers et al. [33], Haberman [34,35], Owadally and Haberman [36] or Haberman et al. [9]. It affirms that the supplementary cost $S C$ is proportional to the unfunded actuarial liability $U A L$, with proportionality constant being generally a positive function of time. This means $C>N C$ if and only if $A L>F$ holds. The supplementary cost obtained in our model contains a modification of this funding procedure, expressed in the second summand of the right-hand term of (33). This term responds to the uncertainty due both to salaries and asset price evolution.

\section{Safe investment}

A special case in the management of the defined benefit pension plan is to consider that the whole wealth of the fund is invested at a safe and constant rate of return. This case is studied here, where the unique source of uncertainty comes from the salaries outgo. The following results can be obtained as a particular case from the previous sections. Our objective is to compare the two investment possibilities.

Now we only consider a bond $P^{0}$ verifying

$$
\mathrm{d} P^{0}(t)=r P^{0}(t) \mathrm{d} t, \quad P^{0}(0)=1,
$$

where $r>0$ is the constant rate of interest.

The evolution of the fund is given by the ODE (ordinary differential equation)

$$
\mathrm{d} F(t)=\left(r F(t)+(\mathbf{u}-\mathbf{k})^{\top} \mathbf{s}\right) \mathrm{d} t, \quad t \geqslant 0,
$$

with initial condition $F(0)=F_{0}>0$.

In the control problem considered here, the only instrument of the manager is the relative rates of contribution $\mathbf{u}$, that we suppose is a measurable adapted process with respect to $\left\{\mathscr{F}_{t}\right\}$ satisfying (5).

The objective functional to be maximized over the class of admissible controls $\mathscr{A}_{t_{0}, F_{0}, \mathbf{s}^{0}}$, is given as in the above section, but since now there is no investment decisions, we write $J\left(\left(t_{0}, F_{0}, \mathbf{s}^{0}\right)\right.$; $\left.\mathbf{u}\right)$ instead of $J\left(\left(t_{0}, F_{0}, \mathbf{s}^{0}\right) ;(\mathbf{u}, \boldsymbol{\Lambda})\right)$. Here, $\mathscr{A}_{t_{0}, F_{0}, \mathbf{s}^{0}}$ is the set of measurable processes $\mathbf{u}$ satisfying (5) and where $F$ and $\mathbf{s}$ satisfy (34) and (13) (without the process $z$ ), respectively. In the above, $\mathrm{E}_{t_{0}, F_{0}, \mathbf{s}^{0}}$ denotes conditional expectation with respect to the initial conditions $\left(t_{0}, F_{0}, \mathbf{s}^{0}\right)$.

The analogous result to Theorem 4.1 is the following.

Theorem 6.1. Suppose that the hypotheses $\mathbf{A}$ and $\mathbf{B}$ hold. Then the optimal relative contribution rates are given by

$$
\tilde{u}_{i}(t, F, \mathbf{s})=k_{i}-\frac{\alpha_{i}}{s_{i}} \alpha^{-\gamma} f^{\gamma}(\mathbf{s}) \tilde{g}^{-\gamma}(t, \mathbf{s}) F, \quad i=1, \ldots, n,
$$

where $\gamma$ and f are defined in (17) and (18), respectively, and where $\tilde{g}:[0, T] \times \mathbb{R}^{n} \longrightarrow \mathbb{R}$ is a positive solution of the non-linear PDE

$$
\begin{aligned}
& g_{t}+(-\rho+r \alpha) g+(1-\alpha) \alpha^{-\gamma \alpha} f^{\gamma}(\mathbf{s}) g^{-\gamma \alpha}+\eta(\mathbf{s})^{\top} g_{\mathbf{s}}+\frac{1}{2} \operatorname{tr}\left\{\beta^{w}(\mathbf{s}) \beta^{w}(\mathbf{s})^{\top} g_{\mathbf{s s}}\right\}=0 \quad \forall(t, \mathbf{s}), \\
& g(T, \mathbf{s})=G^{-\alpha}(\mathbf{s}) \quad \forall \mathbf{s} .
\end{aligned}
$$


At such level of generality it is difficult to compare $\tilde{g}$ with $g$ (the solution of (19) in the presence of risk investment). Similar conclusions as for Proposition 4.1 are obtained with respect to the relative contributions $\mathbf{u}$. Now the fund $F$ satisfies the ODE

$$
\dot{F}(t)=\left(r-\alpha\left(\frac{f(\mathbf{s})}{\alpha \tilde{g}(t, \mathbf{s})}\right)^{\gamma}\right) F(t),
$$

where $\mathbf{s}$ satisfies (13).

Under Hypothesis $\mathbf{C}$ we obtain the following result, analogous to Proposition 5.2.

Proposition 6.1. Suppose that hypotheses $\mathbf{A}, \mathbf{B}$ and $\mathbf{C}$ hold. Then the optimal relative contribution rates are given by

$$
\tilde{u}_{i}(t, F, \mathbf{s})=k_{i}-\frac{\alpha_{i}}{\tilde{h}(t)} \frac{F}{s_{i}}, \quad i=1, \ldots, n,
$$

respectively, where $\tilde{h}$ is given by

$$
\tilde{h}(t)= \begin{cases}\left(\varepsilon^{-\gamma}+\frac{1-\alpha}{\tilde{A}}\right) \mathrm{e}^{\gamma \tilde{A}(T-t)}-\frac{1-\alpha}{\tilde{A}} & \text { if } \tilde{A} \neq 0 \\ \varepsilon^{-\gamma}+T-t & \text { if } \tilde{A}=0\end{cases}
$$

for all $t \in[0, T]$, where the constant $\tilde{A}$ is given by

$$
\tilde{A}=-\rho+r \alpha-\sum_{i=1}^{n} \eta_{i} \alpha_{i}+\frac{1}{2} \sum_{i, j=1}^{n} \sum_{l=m+1}^{m+d} \beta_{i l} \beta_{j l} \alpha_{i} \alpha_{j}+\frac{1}{2} \sum_{i=1}^{n} \sum_{l=m+1}^{m+d} \beta_{i l}^{2} \alpha_{i} .
$$

Analogous observations as those made to Proposition 5.2 apply.

We proceed further to compare the results obtained in this section with those of the section above. For this task we use the properties of the function $h$ established previously and the inequality $A>\tilde{A}$ that obviously holds. Recall that we did introduce the terms high salary growth and moderate salary growth to identify two range of values of the constant $A$, that is always considered negative. A positive value of $A$ would mean a low salary growth when comparing with the size of the source uncertainty. In this case no direct comparison can be made, because $h$ is not monotonic.

1. High salary growth (e.g., directives or employers):

If the mean growth of salaries is such that $A=\max \{A, \tilde{A}\}<-(1-\alpha) \varepsilon^{\gamma}$, then the relative contribution rates when the fund is invested in the bond are lesser than when it is invested in the portfolio, $\tilde{u}_{i}<u_{i}$, for all $i$, which is implied by the inequality $\tilde{h}<h$. Moreover, $E_{F_{0}} \tilde{F}(T)<E_{F_{0}} F(T)$ and $E_{F_{0}} \tilde{\Gamma}(T)<E_{F_{0}} \Gamma(T)$, by (30) and (31), respectively. Thus investment in the mixed portfolio leads the expected fund's level and cumulative income to a higher value than when investment only in the bond is considered. From this observation it follows that the mixed portfolio implies a higher total amortization than investment solely in the bond as it readily follows from the following equalities

$$
\begin{aligned}
\int_{0}^{T}(C(t)-\tilde{C}(t)) \mathrm{d} t & =\int_{0}^{T}(B(t)-\tilde{C}(t)) \mathrm{d} t-\int_{0}^{T}(B(t)-C(t)) \mathrm{d} t \\
& =\left(F_{0}-\tilde{\Gamma}(T)\right)-\left(F_{0}-\Gamma(T)\right)=\Gamma(T)-\tilde{\Gamma}(T) .
\end{aligned}
$$

2. Moderate salary growth (e.g., functionaries or permanent employees):

If the mean growth of the salaries is such that $-(1-\alpha) \varepsilon^{\gamma}<A, \tilde{A}<0$, then $h<\tilde{h}$ and we have $u_{i}<\tilde{u}_{i}$, for all $i$, the opposite conclusion to the item above. At this level of generality, in this case is not possible to compare $E_{F_{0}} \tilde{F}(T)$ with $E_{F_{0}} F(T)$ nor $E_{F_{0}} \tilde{\Gamma}(T)$ with $E_{F_{0}} \Gamma(T)$, and is not possible to recommend the investment in the bond or in the mixed portfolio. 


\section{Conclusions}

We have studied the problem of pension funding in a defined benefit pension plan with $n$ groups of workers from the perspective of a manager who wishes to minimize the relative contributions to plan of the employees and to maximize the terminal relative fund in order to obtain a surplus. We have supposed that all elements of plan are stochastic, including the labor incomes, which are given by $n$ coupled processes and that serve to identify $n$ different groups of workers.

We have found that it is possible to select optimal contributions and portfolio strategies as linear functions of the fund level, depending on all the salaries. Moreover, if the salaries have an exponential form, the optimal contribution made for each group to the fund depends only of its salary, and the optimal investment quantities are independent of salaries, positives, and also proportional to the fund level. We also show in the exponential case that from the aggregated point of view, the manager is implicitly using an amortization method of funding, which is somewhat different to the usual spread method. Furthermore, the form of optimal contributions allows us to compare the case where diversification in investment is contemplated, with that when the manager only invest in a risk-free asset.

Finally, further research can be directed to the numerical solution of the HJB equation in the general case, without supposing exponential salaries.

\section{Appendix A.}

Proof of Theorem 4.1. For the problem of optimal pension funding established in Section 3, the HJB equation is

$$
\begin{aligned}
& -\rho V+V_{t}+\max _{\mathbf{u}, \Lambda}\left\{\prod_{i=1}^{n}\left(k_{i}-u_{i}\right)^{\alpha_{i}}+\left(r F+(\mathbf{u}-\mathbf{k}) \mathbf{s}+\Lambda^{\top}(\boldsymbol{\mu}-r \mathbf{1})\right) V_{F}+\eta^{\top} V_{s}\right. \\
& \left.\quad+\frac{1}{2} \Lambda^{\top} \Sigma \Lambda V_{F F}+\Lambda^{\top} \sigma\left(\beta^{z}\right)^{\top} V_{F \mathbf{s}}+\frac{1}{2} \operatorname{tr}\left\{\beta \beta^{\top} V_{\mathbf{s}}\right\}\right\}=0, \\
& V(T, F, \mathbf{s})=\left(\frac{F}{G(\mathbf{s})}\right)^{\alpha}
\end{aligned}
$$

where $V_{F \mathbf{s}}=\left(V_{F s_{1}}, \ldots, V_{F s_{1}}\right)^{\top}$ and $V_{\mathbf{s s}}$ is the Hessian matrix of $V$ with respect to $\mathbf{s}$.

Let $V$ be a smooth solution of the equation (35), strictly concave with respect to $F$. The maximizers values of the relative contribution rates and the investment rates are given by

$$
\begin{gathered}
\widehat{u_{i}}\left(V_{F}\right)=k_{i}-\frac{\alpha_{i}}{s_{i}} f^{\gamma}(\mathbf{s}) V_{F}^{-\gamma}, \quad i=1, \ldots, n, \\
\widehat{\Lambda}\left(V_{F}, V_{F F}, V_{F \mathbf{s}}\right)=-\Sigma^{-1}(\boldsymbol{\mu}-r \mathbf{1}) \frac{V_{F}}{V_{F F}}-\sigma^{-\top}\left(\beta^{z}(\mathbf{s})\right)^{\top} \frac{V_{F \mathbf{s}}}{V_{F F}},
\end{gathered}
$$

respectively. Note that formula (38) is obtained equating to 0 the partial derivative with respect to $\lambda_{i}$ in the expression between brackets of (35). In order to obtain the expression (37) we proceed analogously with $u_{i}$ to arrive at

$$
-\frac{\alpha_{i}}{k_{i}-u_{i}} \prod_{j=1}^{n}\left(k_{j}-u_{j}\right)^{\alpha_{j}}+s_{i} V_{F}=0, \quad i=1, \ldots, n .
$$

Dividing the equation $i$ by the equation corresponding to e.g. $i=1$, we obtain

$$
u_{i}=k_{i}-\frac{\alpha_{i}}{\alpha_{1}} \frac{s_{1}}{s_{i}}\left(k_{1}-u_{1}\right), \quad i=1, \ldots, n,
$$

and substituting this expression in (39) for $i=1$ results in

$$
u_{1}=k_{1}-\frac{\alpha_{1}}{s_{1}} f^{\gamma}(\mathbf{s}) V_{F}^{-\gamma}
$$


with $\gamma$ and $f$ given by (17) and (18), respectively. We have used the fact $\alpha=\sum_{i=1}^{n} \alpha_{i}$. Finally, substituting (41) in (40) we have (37).

Once we have substituted the values for $\widehat{u_{i}}$ and $\widehat{\Lambda}$ in (35), the structure of the equation obtained

$$
\begin{gathered}
-\rho V+V_{t}+(1-\alpha) f^{\gamma}(\mathbf{s}) V_{F}^{-\gamma \alpha}+r F V_{F}-\frac{1}{2} \boldsymbol{\theta}^{\top} \boldsymbol{\theta} \frac{V_{F}^{2}}{V_{F F}}-\frac{V_{F}}{V_{F F}} V_{F \mathbf{s}}^{\top} \beta^{z}(\mathbf{s}) \boldsymbol{\theta} \\
+\eta^{\top} V_{\mathbf{s}}-\frac{1}{2 V_{F F}} V_{F \mathbf{s}}^{\top} \beta^{z}(\mathbf{s})\left(\beta^{z}(\mathbf{s})\right)^{\top} V_{F \mathbf{s}}+\frac{1}{2} \operatorname{tr}\left\{\beta(\mathbf{s}) \beta(\mathbf{s})^{\top} V_{\mathbf{s s}}\right\}=0,
\end{gathered}
$$

suggests a function of separated variables of $(F, \mathbf{s})$ and homogeneous in $F$ of degree $\alpha$ as the solution

$$
\widehat{V}(t, F, \mathbf{s})=g(t, \mathbf{s}) F^{\alpha} .
$$

Imposing this solution, the function $g$ is characterized by equation (19) together with the final condition (20), which follows from (36). Finally (15) and (16) are found from substitution of $\widehat{V}$ in (37) and (38), respectively.

Proof of Proposition 5.1. The direct proof of present result is immediate, it is sufficient to check that $g$, given by (23), satisfies (19)-(20). We prefer, however, give a more constructive proof.

For it we prove, in a first step, that $g$ can be expressed as the product of a function only depending on $t$ and the known function $f$, given in (18). So we postulate: $g(t, \mathbf{s})=a(t) f(\mathbf{s})$, where $a: \mathbb{R} \longrightarrow \mathbb{R}$ is a function only time-dependent. The following properties are verified:

$$
g_{t}=\dot{a} f, \quad g_{\mathbf{s}}=a f_{\mathbf{s}}, \quad g_{\mathbf{s s}}=a f_{\mathbf{s s}} .
$$

Substituting in (19) we obtain

$$
\begin{aligned}
f \dot{a} & +\left(-\rho+r \alpha+\frac{1}{2} \frac{\alpha}{1-\alpha} \boldsymbol{\theta}^{\top} \boldsymbol{\theta}\right) f a+(1-\alpha) \alpha^{-\gamma \alpha} f a^{-\gamma \alpha}+\eta(\mathbf{s})^{\top} f_{\mathbf{s}} a \\
& -\alpha f_{\mathbf{s}}^{\top} \beta^{z}(\mathbf{s}) \boldsymbol{\theta} a+\frac{1}{2} \frac{\alpha}{f} f_{\mathbf{s}}^{\top} \beta^{z}(\mathbf{s})\left(\beta^{z}(\mathbf{s})\right)^{\top} f_{\mathbf{s}} a+\frac{1}{2} \operatorname{tr}\left\{\beta(\mathbf{s}) \beta(\mathbf{s})^{\top} f_{\mathbf{s s}}\right\} a=0 \quad \forall(t, \mathbf{s}) .
\end{aligned}
$$

Applying the properties of $f$,

$$
f_{s_{i}}=-\frac{\alpha_{i}}{s_{i}} f, \quad f_{s_{i} s_{j}}= \begin{cases}\left(1+\alpha_{i}\right) \frac{\alpha_{i}}{s_{i}^{2}} f & \text { if } i=j, \\ \frac{\alpha_{i} \alpha_{j}}{s_{i} s_{j}} f & \text { if } i \neq j,\end{cases}
$$

for all $i, j=1, \ldots n$, and using Hypothesis $\mathbf{C}$, we arrive to the Bernoulli equation

$$
\dot{a}+A a+(1-\alpha) \alpha^{-\gamma \alpha} a^{-\gamma \alpha}=0,
$$

where $A$ is given by (25). Taking into account (20) we obtain the final condition for function $a$ :

$$
a(T)=\frac{1}{\prod_{i=1}^{n} \alpha_{i}^{\alpha_{i}}} .
$$

In order to prove (23), the second step is to make the change of variable: $a(t)=\alpha^{-\alpha} h^{1-\alpha}(t)$. The differential equations (42)-(43) in the new function $h$,

$$
\begin{aligned}
& \dot{h}+\frac{A}{1-\alpha} h+1=0, \\
& h(T)=\frac{\alpha^{\gamma \alpha}}{\prod_{i=1}^{n} \alpha_{i}^{\gamma \alpha_{i}}}=\varepsilon^{-\gamma},
\end{aligned}
$$

is of linear type and of first order. Therefore, we can solve it analytically, obtaining (24).

Proof of Proposition 5.2. Substituting (23) in (15) and (16) we obtain (26) and (27), respectively. 


\section{References}

[1] Boulier JF, Huang S, Taillard G. Optimal management under stochastic interest rates: the case of a protected defined contribution pension fund. Insurance: Mathematics and Economics 2001;28:173-89.

[2] Vigna E, Haberman S. Optimal investment strategy for defined contribution pension schemes. Insurance: Mathematics and Economics 2001;28:233-62.

[3] Haberman S, Vigna E. Optimal investment strategies and risk measures in defined contribution pension schemes. Insurance: Mathematics and Economics 2002;31:35-69.

[4] Deelstra G, Grasselli M, Koehl PF. Optimal investment strategies in a CIR model. Journal of Applied Probability 2000;37:936-46.

[5] Deelstra G, Grasselli M, Koehl PF. Optimal investment strategies in the presence of a minimum guarantee. Insurance: Mathematics and Economics 2003;33:189-207.

[6] Battocchio P, Menoncin F. Optimal pension management in a stochastic framework. Insurance: Mathematics and Economics 2002;34:79-95.

[7] Haberman S, Sung JH. Dynamics approaches to pension funding. Insurance: Mathematics and Economics 1994;15:151-62.

[8] Chang SC. Optimal pension funding through dynamic simulations: the case of Taiwan public employees retirement systems. Insurance: Mathematics and Economics 1999;24:187-99.

[9] Haberman S, Butt Z, Megaloudi C. Contribution and solvency risk in a defined benefit pension scheme. Insurance: Mathematics and Economics 2000;27:237-59.

[10] Taylor G. Stochastic control of funding systems. Insurance: Mathematics and Economics 2002;30:323-50.

[11] Chang SC, Tzeng LY, Miao JCY. Pension funding incorporating downside risks. Insurance: Mathematics and Economics 2003;32:217-28.

[12] Josa-Fombellida R, Rincón-Zapatero JP. Minimization of risks in pension funding by means of contribution and portfolio selection. Insurance: Mathematics and Economics 2001;29:35-45.

[13] Josa-Fombellida R, Rincón-Zapatero JP. Optimal management of risks in defined-benefit stochastic pension funds. Insurance: Mathematics and Economics 2004;34:489-503.

[14] Cairns AJG. Some notes on the dynamics and optimal control of stochastic pension fund models in continuous time. ASTIN Bulletin 2000;30: $19-55$.

[15] Merton RC. Lifetime portfolio selection under uncertainty: the continuous-time case. The Review of Economics and Statistics 1969;51: 247-57.

[16] Merton RC. Optimal consumption and portfolio rules in a continuous-time model. Journal of Economic Theory 1971;3:373-413.

[17] Bowers NL, Gerber HU, Hickman JC, Jones DA, Nesbitt CJ. Actuarial Mathematics. Illinois: The Society of Actuaries; 1997.

[18] O’Brien T. A stochastic-dynamic approach to pension funding. Insurance: Mathematics and Economics 1986;5:141-6.

[19] O'Brien T. A two-parameter family of pension contribution functions and stochastic optimization. Insurance: Mathematics and Economics 1987;6:129-34.

[20] Berkelaar A, Kouwenberg R. Retirement saving with contribution payments and labor income as a benchmark for investments. Journal of Economic Dynamics and Control 2003;27:1069-97.

[21] Koo HK. Consumption and portfolio selection with labor income: a continuous time approach. Mathematical Finance 1998;8:49-65.

[22] Duffie D, Zariphopoulou T. Optimal investment with undiversifiable income risk. Mathematical Finance 1993;3:135-48.

[23] Duffie D, Fleming W, Soner M, Zariphopoulou T. Hedging in incomplete markets with HARA utility. Journal of Economic Dynamics and Control 1997;21:753-82.

[24] Cairns AJG, Blake D, Dowd K. Stochastic lifestyling: optimal dynamic asset allocation for defined contribution pension plans. Journal of Economic Dynamics and Control 2006, Forthcoming.

[25] Battocchio P, Menoncin F. Optimal portfolio strategies with stochastic wage income and inflation: the case of a defined contribution pension plan. Working Paper CeRP No. 19-02, Torino; 2002.

[26] Zariphopoulou T. Consumption-investment models with constraints. SIAM Journal on Control and Optimization 1994;32:59-85.

[27] Grossman SJ, Laroque G. Asset pricing and optimal portfolio choice in the presence of illiquid durable consumption goods. Econometrica 1990;58:25-51.

[28] Damgaard A, Fuglsbjerg B, Munk C. Optimal consumption and investment strategies with a perishable and an indivisible durable consumption good. Journal of Economic Dynamics and Control 2003;28:209-53.

[29] Fleming WH, Soner HM. Controlled Markov processes and viscosity solutions. New York: Springer; 1993.

[30] Duffie D. Dynamic asset pricing theory. 3rd edition, Princeton, NJ: Princeton University Press; 2001.

[31] Karatzas I, Shreve SE. Methods of mathematical finance. New York: Springer; 1998.

[32] Arnold L. Stochastic differential equations. Theory and applications. New York: Wiley; 1974.

[33] Bowers NL, Hickman JC, Nesbitt CJ. The dynamics of pension funding: contribution theory. Transactions of the Society of Actuaries 1979;31: 93-119.

[34] Haberman S. Pension funding. The effect of changing the frequency of valuations. Insurance: Mathematics and Economics 1993;13:263-70.

[35] Haberman S. Stochastic investment returns and contribution rate risk in a defined benefit pension scheme. Insurance: Mathematics and Economics 1997;19:127-39.

[36] Owadally MI, Haberman S. Pension fund dynamics and gains/losses due to random rates of investment return. North American Actuarial Journal 1999;3:105-17. 